\title{
TEACHING THE CLOUD: SECURITY REMAINS A CONCERN FOR INFORMATION TECHNOLOGY STUDENTS
}

\author{
John J. Scarpino, Pittsburgh Technical College, scarpino.john@ptcollege.edu \\ Michael Hodder, Pittsburgh Technical College,hodder.michael@ptcollege.edu \\ Gary A. Davis, Robert Morris University, davis@rmu.edu \\ Paul Kovacs, Robert Morris University, kovacs@rmu.edu \\ Theophilus D. Owusu, Keiser University, towusu@keiseruniversity.edu \\ Matthew R. Kisow, NSABP Foundation, matthew.kisow@nsabp.org
}

\begin{abstract}
Courses in Cloud Computing in higher education are in high demand, as industry embraces this technology. The main author of the paper developed a class in cloud computing. The topics of this course were then sent to two institutions of higher education in an effort to determine which of these topics has the strongest demand from students who take cloud computing courses. The survey responses were solicited from associate, bachelors, masters and doctoral-level students. This research assists and aides others who are planning to implement a Cloud Computing class within their curriculum.
\end{abstract}

Keywords: Cloud Computing, Higher-Ed Courses, Cloud Security, IT Curriculum

\section{INTRODUCTION}

Cloud Computing is an Internet-based computing system that provides up-to-date, on-demand services for administration and practice of large shared resources including storage, data, and infrastructures. Cloud Technologies, especially the MapReduce framework and its open-source employment of Hadoop or Hyper-V and VMware, permits scalable distributed processing of enormous quantities of data (Chen, Gallagher, Pailthorpe, \& Li, 2012). To grow and administer information systems for large scale data analysis and processing, cloud computing knowledge and skills are crucial and required for computing specialists.

The formal definition of cloud computing comes from the National Institute of Standards and Technology (NIST), defines Cloud Computing as “ . . . a model for enabling ubiquitous, convenient, on-demand network access to a shared pool of configurable computing resources (e.g., networks, servers, storage, applications and services) that can be rapidly provisioned and released with minimal management effort or server provider interaction" (Mell \& Grance, 2011 p.1).

\section{RELATED TRENDS AND STUDIES}

Changchit (2015), notes that technology acts as a driving force for the development of monetary systems and value of life. As IT continues to mature and become a large part of the world. Worldwide, individuals are acquiring new skills in order to accomplish their needs. Two major trends in IT are Big Data and Cloud Computing. These two trends are closely related, as investments in Big Data tend to require increased data storage; which, in turn, requires increased investments in Cloud Computing. Cloud computing, therefore, has garnered much attention in both practical and theoretical settings. There are many benefits involved when migrating to cloud computing; it is cost effective and sometimes, even free. The cost, however, depends on the service areas desired. The costs of IT are reduced because there is no connecting, maintaining, or upgrading of software. Costs can also be reduced as a result of minimized security risks and reduction in overtime (Changchit, 2015).

According to the CDW Corporations annual report (Campbell, 2009), a survey of 1,242 information technology decision makers found that the number of organizations that adopt or plan to adopt cloud computing has been increasing, compared to the annual report of 2011. The reasons indicated for this increase are as follows: $55 \%$ increase in efficiency, a $49 \%$ improvement in mobility, a $32 \%$ increase in the ability to innovate, a $31 \%$ increase in available IT staff for other projects, a $25 \%$ reduction in IT operating costs, and a $24 \%$ increase in the ability to offer 
new products/services. The CDW report also revealed that $43 \%$ of higher-education institutions are implementing or maintaining cloud computing. This number has increased by $10 \%$, compared to $34 \%$ in 2011 (Campbell, 2009). Finally, the CDW found that the specific applications that universities were moving to the Cloud are: 1) Storage (31\%), 2) Messaging/conferencing and collaboration (29\%), and 3) Computing power (25\%).

Advanced educational facilities and assets are now accessible to aid students in developing a complete appreciation of "The Cloud." The addition of cloud-based assets into the technology data centers of organizations of all sizes is increasing rapidly. The scope of the cloud computing arena was estimated to increase to 18.5 percent in 2013 by reaching a total of $\$ 131$ billion worldwide. In addition, Gartner forecasts that $\$ 677$ billion will be consumed worldwide on cloud services from 2013 to 2016, within the North American market, including 59 percent of new spending (Mitchell \& Meggison, 2014). Based on cloud service providers, organizations can receive all-inclusive IT amenities that provide tactical sustenance and result in operative productivities. Pratt (2012) showed that business intelligence and cloud computing are two of the emergent technology movements that IT managers assess as top subjects for years to come; yet the topics have not yet made their way into college curricula (Mitchell \& Meggison, 2014).

Even though higher education has been reluctant to formally incorporate Cloud Computing topics into their curricula, many top universities have offered cloud-associated subjects. Some schools have even combined Cloudbased technologies, like Hadoop, Hyper-V or VMware, into their programs. More than a few schools have integrated Hadoop into their curricula. The University of California at Berkley, for example, has combined four, two-week long Hadoop projects into an introductory machine computing course (Fox, 2009). St. Olaf College has built systems to run Hadoop based on old and unused computers for students in computer science courses. St. Olaf then built virtual systems from lab machines for parallel and distributed computing courses (Brown, 2009). Dr. Wang (2011) at Michigan Technological University presented cloud computing material within a senior capstone project for IT undergraduate students. A number of schools are currently using cloud technologies to improve students' learning experiences. For example, Louis M. Vaquero of Hewlett Packard Labs in Bristol, United Kingdom did a high quality case study linking cloud services in an advanced computer science course (Chen et al., 2012).

There have also been specific undergraduate and graduate-level courses offered on Cloud Computing. A course by Lee Gillam (2012) at Surrey University is one of the first offered of its kind. Gillam's course is a general introductory course in cloud computing that starts by introducing the concepts of software, platform, and infrastructure as a service. It also covers relevant cloud protocols, such as SOAP and REST, and discusses the MapReduce computational model in relation to Hadoop. The course touches on grid and peer-to-peer computing, and discusses service-level agreements, cloud economics, and security. Finally, the American Military Academy (2016) offers a Certificate in Cloud Computing. The topics included in the certificate involve data security, data governance, compliance, and legacy application migration issues.

Whether in industry or education, cloud computing attempts to regulate Information Technology (IT) costs and promotes technology agility. Therefore, organizations are applying cloud computing services at an amazing rate. Predicted job growth within cloud computing worldwide is 13.8 million by 2015 . The growth will predominantly involve small- and medium-sized businesses. As a result, students should have a more complete knowledge base of this vital aspect of current skills in cloud computing (i.e., students need to utilize cloud computing for more than just virtualization). Incorporating cloud computing theories and practice into the IT curriculum allows expertise and skill growth that is related directly to today's IT industry. Courses in cloud computing impact many educational topics, including business law, computer applications, economics, and management of information systems (Mitchell \& Meggison, 2014).

\section{LIMITATIONS OF STUDY}

Participants in the current study were a combination of Associate, Bachelor, Master, and Doctoral-level students. The results reported throughout the study reflect the responses provided by participants at all of these educational 
levels. Even though this study sought to establish general perceptions of students, the results may be more generalizable if stratified by educational level. To address this limitation, the current authors plan to report results by educational level in a follow-up study.

\section{RESEARCH QUESTIONS}

The purpose of this paper is to identify those topics that are most important to associate, bachelors, masters and doctoral-level students in IT programs. The value of this information may allow educators to focus on those cloud computing topics that are most relevant to students.

Our research was centered upon the following four research questions:

1. Which Cloud Computing topic do IT students rank as most interesting?

2. What do IT students rank as being the biggest inhibiting factor to the use of Cloud Computing?

3. Which methods/techniques do IT students feel are most interesting for teaching Cloud-Computing?

4. What topics do IT students feel should be added to Cloud-Computing curricula?

\section{RESEARCH METHODOLOGY}

This study involved an online survey instrument that consisted of 11 closed-ended questions and was administered to students enrolled in CIS courses at two institutions of higher education. One school was a private university. The other school was a technical institute. Both schools were medium-sized institutions. The students at the private university consisted of undergraduate, graduate, and post-graduate students. The students at the technical institute consisted only of associate-level, undergraduate students. A total of 144 students responded to the survey. The respondents used QuestionPro survey software to submit their results directly into an electronic database for analysis. The survey was active from February 1, 2016 to March 12, 2016.

\section{RESULTS}

A total of 144 students completed the survey. Of the $144,89(61.81 \%)$ of the students were from the private university. The remaining $55(38.19 \%)$ of the students were from the technical institute. Overall, out of the 144 student who responded to the survey, $52(36.11 \%)$ were at the Associate's Degree-level, $33(22.92 \%)$ were at the Bachelor's Degree-level, 30 (20.83\%) were at the Master's Degree-level, 24 (16.67\%) were at the Doctoral Degreelevel, and $5(3.47 \%)$ reported "Other" as their education level.

As stated previously, the first research question was, "Which Cloud Computing topic do IT students rank as most interesting?" In order to answer this question, respondents were asked to rank-order ten popular topics typically found in cloud computing curricula. The results from this question are shown in Table 1: Ranking of Cloud Topic Interest. 
Table 1. Rankings of Cloud Topic Interest

\begin{tabular}{|l|c|c|c|}
\hline \multicolumn{1}{|c|}{ Topics } & Avg. Ranking & Count & Percentage \\
\hline Security, Risk, \& Assurance of the Cloud & 4.54 & 23 & $15.97 \%$ \\
\hline Design \& Planning of the Cloud & 5.02 & 7 & $4.86 \%$ \\
\hline Changes, Innovation, \& Evolution of the Cloud & 5.05 & 13 & $9.03 \%$ \\
\hline Administration \& Management of the Cloud & 5.29 & 22 & $15.28 \%$ \\
\hline Cloud Computing Features \& Theories & 5.30 & 7 & $4.86 \%$ \\
\hline Intro / Overview - What is the Cloud? & 5.70 & 43 & $29.86 \%$ \\
\hline Enterprise \& Professional Need for the Cloud & 5.80 & 4 & $2.78 \%$ \\
\hline Future / Next Steps in Cloud Computing & 5.80 & 12 & $8.33 \%$ \\
\hline Software \& Data Deployment to the Cloud & 6.15 & 6 & $4.17 \%$ \\
\hline The Cloud Platform (SaaS, IaaS, PaaS, iPaaS) & 6.35 & 7 & $4.86 \%$ \\
\hline
\end{tabular}

As shown in Table 1, the topic with the highest level of interest (i.e., 1 = "most interesting," $10=$ "least interesting") was Security, Risk, and Assurance of the Cloud (average rank 4.54). The topic with the second highest level of interest was Design \& Planning of the Cloud (average rank 5.02). Finally, the topic with the third highest level of interest was Changes, Innovation and Evolution of the Cloud (average rank 5.05). It is also interesting to note from Table 1 that $43(29.86 \%)$ of the respondents ranked Introduction/Overview - What is the Cloud? as the number one ranked topic, in terms of interest. More specifically, a third of the respondents would consider an introduction to the cloud as an interesting class topic. This result suggests that, even though cloud security is a concern, many students still need a basic introduction to what cloud computing entails.

The second research question was, "What do IT students rank as being the biggest inhibiting factor to the use of Cloud Computing?" In order to answer this question, respondents were asked to rank-order ten inhibiting factors regarding cloud computing. The results from this question are shown in Table 2: Ranking of Cloud Inhibiting Factors.

Table 2. Ranking of Cloud Inhibiting Factors

\begin{tabular}{|l|c|c|}
\hline \multicolumn{1}{|c|}{ Factors } & Count & Percentage \\
\hline Security Concerns & 112 & $24.51 \%$ \\
\hline Integration with Current Networks, Applications, \& Systems & 62 & $13.57 \%$ \\
\hline Legal \& Regulatory Compliance & 49 & $10.72 \%$ \\
\hline Implementing Policies to Move Applications to the Cloud & 40 & $8.75 \%$ \\
\hline System Performance / Availability & 38 & $8.32 \%$ \\
\hline Lack of Standards Among Cloud Service Providers & 36 & $7.88 \%$ \\
\hline Deciding Which Applications to Move to the Cloud & 34 & $7.44 \%$ \\
\hline Lack of Visibility of Future Cost & 31 & $6.78 \%$ \\
\hline Difficulty Finding Vendors with Needed Skillset / Offerings & 25 & $5.47 \%$ \\
\hline Managing a Vendor / Partner & 18 & $3.94 \%$ \\
\hline This Question is Not Applicable to Me & 11 & $2.41 \%$ \\
\hline Other & 1 & $0.22 \%$ \\
\hline
\end{tabular}

As shown in Table 2, the inhibiting factor with the highest ranking (i.e., $1=$ "most inhibiting," $10=$ "least inhibiting") was Security Concerns. Specifically, 112 (24.51\%) of the respondents ranked Security Concerns as the most inhibiting factor to implementing cloud computing. In addition, $62(13.57 \%)$ of the respondents ranked Integration with Current Networks/Applications/Systems as the second most inhibiting factor to implementing cloud computing. Finally, $49(10.72 \%)$ of the respondents ranked Legal and Regulatory Compliance as the third most inhibiting factor to the implementation of cloud computing. In addition, 11 of the respondents indicated that the question was Not Applicable to him/her. Lastly, one respondent chose the category Other and entered "Cost Structure" as the factor most inhibiting to the implementation of cloud computing. 
The third research question asked, "Which methods/techniques do IT students feel are most interesting for teaching Cloud-Computing?" In order to answer this question, respondents were presented with the following possible teaching methods/techniques: 1) Lab Activities, 2) Assignments, 3) Exams, 4) Videos, 5) Discussions, 6) Individual Projects, and 7) Group Projects. The respondents were also asked to select Other and to enter a proposed teaching technique. As expected, $112(25.4 \%)$ of the respondents chose Lab Activities as the most interesting teaching method/technique for cloud computing. Group Projects were selected as the most interesting method/technique by $74(16.78 \%)$ of the respondents. Finally, Individual Projects were selected as the most interesting method/technique by $66(14.97 \%)$ of the respondents. A breakdown of all teaching methods/techniques are shown in Table 3: Teaching Methods for Cloud Computing.

Table 3. Teaching Methods for Cloud Computing

\begin{tabular}{|l|c|c|}
\hline \multicolumn{1}{|c|}{ Teaching Methods } & Count & Percentage \\
\hline Lab Activities & 112 & $25.4 \%$ \\
\hline Group Projects & 74 & $16.78 \%$ \\
\hline Individual Projects & 66 & $14.97 \%$ \\
\hline Discussions & 63 & $14.29 \%$ \\
\hline Assignments & 60 & $13.61 \%$ \\
\hline Videos & 50 & $11.34 \%$ \\
\hline Examinations & 15 & $3.40 \%$ \\
\hline Other & 1 & $0.23 \%$ \\
\hline
\end{tabular}

Finally, the last research question asked, "What topics do IT students feel should be added to Cloud-Computing curricula?" In order to answer this question, the respondents were presented with a list of ten topics regarding cloud computing. The respondents were asked to review the list and add any additional topics they feel should be included in a class on cloud computing. Out of 144 respondents, 137 indicated that they did not wish to add any additional topics to the list. Seven of the respondents, however, provided additional topics to be included in a cloud computing class. The list of the ten existing cloud computing topics are shown in Table 4: Additional Cloud Computing Topics. Table 4 also shows the seven additional topics that were provided by the survey respondents.

Table 4. Additional Cloud Computing Topics

\begin{tabular}{|l|l|}
\hline \multicolumn{1}{|c|}{ Existing Topics } & \multicolumn{1}{c|}{ Additional Topics } \\
\hline Administration \& Management of the Cloud & Admin \& Management of the Cloud \\
\hline Changes, Innovations, \& Evolution of the Cloud & Rapid provisioning and disestablishment of cloud services \\
\hline Cloud Computing Features \& Theories & Hands-on of setup, configuration, and use \\
\hline Design \& Planning of the Cloud & Cloud cost and benefit evaluation \\
\hline Enterprise / Professional Need for Cloud Computing & Economics of Cloud \\
\hline Future / Next Steps in Cloud Computing & Invention of the Cloud \\
\hline Introduction / Overview - What is the Cloud? & Cloud Computing in Hardware \\
\hline Security, Risk, \& Assurance of the Cloud & \\
\hline Software \& Data Deployment to the Cloud & \\
\hline The Cloud Platform (SaaS, IaaS, PaaS, iPaaS) & \\
\hline
\end{tabular}

\section{CONCLUSIONS}

This study was undertaken to determine ways that cloud computing topics can be most effectively incorporated into higher education curricula. The study also sought to determine the best teaching methods for the instruction of cloud computing. The results of the study are both remarkable and practical for academia.

Specifically, it is clear from the results that security holds the biggest interest and concern among college students. It is also clear that students recognize the inherent security threats that cloud computing could pose to industry and commerce. The findings of the current study are consistent with other related research in the field of cloud 
computing. For example, Tout, Sverdlik and Lawver (2009) found that the primary concern that cloud computing adopters have is the security of enterprise information. Data placed in storage clouds can potentially be located in, and sent across communication channels of a totally different country. Those countries may have significantly different data privacy laws, and therefore, may expose sensitive data to unauthorized access. In retrospect, the security risks associated with cloud computing is not much different than the risks associated with traditional outsourcing: in both situations, sensitive data can be exposed to the risks of a global computing environment. As the volume of global information continues to increase, the security challenges of Cloud Computing, and other new technologies, will remain a consistent challenge to the Information Technology professional.

In terms of teaching methods and techniques, it was found that Lab Activities, followed by Group Projects and Individual Projects were ranked as most interesting for teaching cloud computing. From these results, it appears that students want an applied, hand-on approach to cloud computing instruction.

Furthermore, the additional topics that students felt should be added to cloud computing curricula indicate a desire for a micro- and macro- understanding of how cloud computing impacts management, administration, and costs. In addition, it is clear from the study that students want an understanding of the overall economic impact of implementation, and upkeep of cloud computing.

Finally, the study provided insight into the respondents' basic knowledge of cloud computing. Even though security concerns had the highest average ranking for importance, the largest number of respondents (i.e., nearly one third) selected Introduction/Overview - What is the Cloud? as the most interesting topic in cloud computing. These results suggest that an Introduction to Cloud Computing course may alleviate some cloud security concerns among today's college students.

\section{REFERENCES}

Brown, R. A. (2009). Hadoop at Home: Large-scale Computing at a Small College, SIGCSE Bull., 41(1), pp. 106110 .

Campbell, Steve. (2009). TimeSharing 2.0. HPC Wire. Retrieved from: http://www.hpcwire.com/hpcwire/200911-03/timesharing_2_0.html

Changchit, C. (2015). Cloud computing: should it be integrated into the curriculum? International Journal of Information and Communication Technology Education, 11(2), 105-117.

Chen, L., Liu, Y., Gallagher, M., Pailthorpe, B., Sadiq, S., Shen, H., \& Li, X. (2012) Introducing cloud computing topics in curricula. Journal of Information Systems Education, 23(3). 315-324.

Cloud Computing: Undergraduate Certificate (2016). American Military Academy. Retrieved from http://www.amu.apus.edu/academic/schools/science-technology-engineering-and-math/certificateug/cloud-computing.html

Fox, A. (2009). Cloud computing in education. Retrived March 6, 2015 from https://inews.berkeley.edu/articles/Spring2009/cloud-computing

Gillam, L., Li, B. \& O'Loughlin, J. Teaching clouds: lessons taught and lessons learnt. Cloud Computing for Teaching and Learning: Strategies for Design and Implementation. Ed. Lee Chao. IGI Global, 2012.

Mell, P., \& Grance, T. (2011).The NIST definition of cloud computing. National Institute of Standards and Technology. Available from: http://csrc.nist.gov/publications/nistpubs/800-145/SP800-145.pdf

Mitchell, R., \& Meggison, P. (2014). Strategies for integrating cloud computing concepts. Journal of Applied Research for Business Instruction, 12(2). 


\section{Issues in Information Systems}

Volume 17, Issue II, pp. 135-141, 2016

Pratt, M. K. (2012). Stressing over skills. Computerworld, 46(7), 26-27.

Tout, S., Sverdlik, W., \& Lawver, G. (2009). Cloud computing and its security in higher education. ISECON, 26(1), $1-5$.

Wang, X., Hembroff, G. C., Cerier, A. B. and Perrault, B. W. (2011) Introducing Cloud Computing with a Senior Design Project in Undergraduate Education of Computer System and Network Administration, Proceedings of the 2011 Conference on Information Technology Education, West Point, New York, pp. 177-182. 